\title{
Correction of Carpal Valgus and Flexural Deformity with z-Tenotomy and Anastomosis Procedure in a Dog
}

\author{
Mikaela Gondolfe $^{1} \quad$ Mark Garneau ${ }^{1}$ \\ ${ }^{1}$ Department of Surgery, Veterinary Specialty Centre of Seattle, \\ Lynnwood, Washington, United States \\ VCOT Open 2020;3:e28-e32.
}

\begin{abstract}
Address for correspondence Mikaela Gondolfe, DVM, Department of Surgery, Veterinary Specialty Centre of Seattle, 20115 44th Avenue, Lynnwood, WA 98036, United States

(e-mail: mgondolfe@gmail.com).
\end{abstract}

\begin{abstract}
Keywords

- carpal valgus

- flexural deformity

- z-tenotomy

This study aimed to describe a staged bilateral z-tenotomy and anastomosis procedure of flexor carpi ulnaris and superficial digital flexor tendons for the correction of carpal valgus and flexural deformity in a 1-year-old male-neutered Akita dog. Bilateral carpal valgus and flexural deformity were observed with palpably taut flexor carpi ulnaris tendons. The dog had a mild, weight-bearing bilateral forelimb lameness with the left forelimb more severely affected. Both forelimbs were treated with staged z-tenotomy and anastomosis procedure of both flexor carpi ulnaris and superficial digital flexor tendons. Successful tendon lengthening and correction of carpal valgus were achieved via z-tenotomy and anastomosis of affected tendons. No complications were observed. An 18-month follow-up revealed no evidence of lameness or carpal valgus. Though tendon injuries commonly occur in small animal patients, there is a shortage of reported cases, especially involving musculotendinous contractures. There are even fewer reports of successful tenotomy procedures. This case report supports successful outcome of the z-tenotomy procedure in a canine patient.
\end{abstract}

\section{Introduction}

Muscle contracture consists of fibrosis of the muscle leading to increased resistance and inability for the muscle to stretch, which results in a shortened tendon or muscle. ${ }^{1}$ Underlying causes include trauma, infectious agents, fracture, compartment syndrome, primary myopathies or neoplasia. ${ }^{2}$ In the case of flexor carpi ulnaris contracture, it is more commonly reported in puppies aged 6 to 24 weeks. ${ }^{3,4}$ With flexor carpi ulnaris contracture, a limb deformity develops characterized by a flexed carpus with inability to extend. In younger animals, it often resolves spontaneously, but can be treated with carpal support bandages. ${ }^{4-6}$ Exact pathogenesis of flexor carpi ulnaris tendon contracture is unknown. Musculotendinous contractures occur in small animal patients; however, reports in the literature are scarce. ${ }^{4-9}$ This case report provides documentation of a successful tendon lengthening procedure and correction of carpal valgus for the treatment of flexor carpi ulnaris and superficial digital flexor tendon contracture.

received

December 22, 2019

accepted after revision

March 15, 2020

\section{Clinical Report}

\section{History and Timeline}

A 1-year-old male neutered Akita with a history of a left medial patellar luxation (LMPL) corrective surgery presented to Veterinary Specialty Centre of Seattle Surgery Department for repair of his right medial patellar luxation (RMPL). The previous LMPL repair had been performed at 10 months of age after presenting for an audible popping sound and bilateral pelvic limb lameness. A recession trochleoplasty, tibial tuberosity transposition and lateral imbrication had been performed for surgical correction of the LMPL. Medical history included being fully vaccinated, and obtained by the owner as a puppy with no history of medical illness associated with litter mates. Home environment involved living with one other dog with appropriate flooring and space for activity. At the time of presentation for the RMPL repair, the owner noticed a recent onset of left forelimb lameness of 2 weeks duration. On physical examination, the dog had moderate bilateral carpal valgus and carpal hyperflexion with more severe changes noted on

\footnotetext{
(c) 2020 Georg Thieme Verlag KG Stuttgart · New York
}

License terms

10.1055/s-0040-1710069. ISSN 2625-2325.

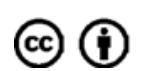




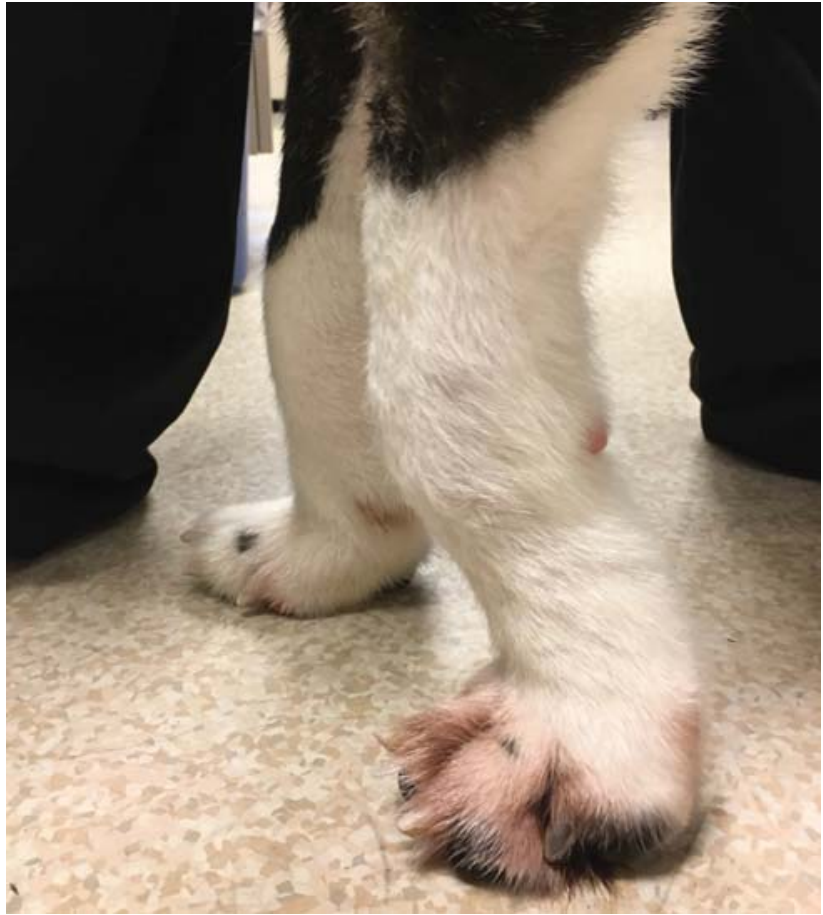

Fig. 1 Lateral view of carpal valgus exhibited on initial physical examination.

the left forelimb (-Figs. 1 and 2). The dog was ambulatory with grade I/IV right hindlimb and left forelimb lameness. There was a right grade II/IV MPL. Radiographs of the forelimbs were consistent with physical examination findings (bilateral carpal valgus and hyperflexion) with no evidence of bone deformity or physeal irregularities. The RMPL was surgically

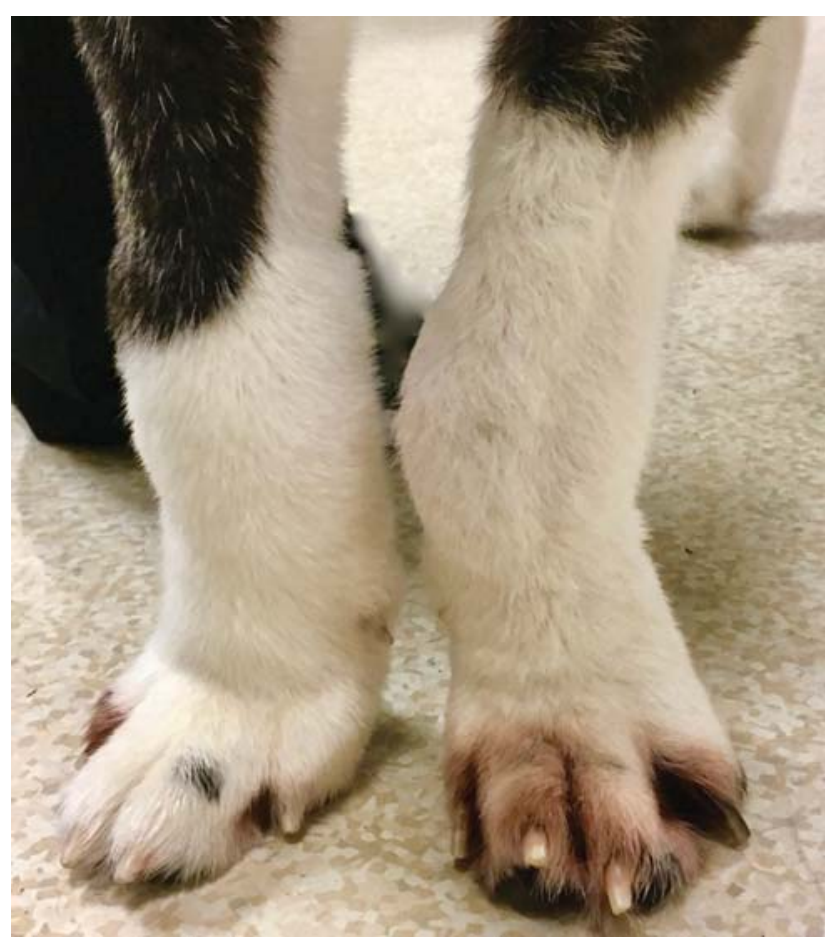

Fig. 2 Frontal view of bilateral carpal valgus with more severity visible in the left forelimb. corrected, and 2 weeks postoperatively, the owner noted worsening of the left forelimb lameness. Orthopaedic examination at that time showed a grade II/IV lameness on left forelimb and right hindlimb. The left carpal valgus and carpal hyperflexion had worsened over this time period. The left flexor carpi ulnaris tendon palpated more taut than the right. Given the physical examination findings, flexor carpi ulnaris contracture was suspected. It was discussed that muscle fibrosis may be present, leading to inability of the tendon to stretch, and surgical correction may be needed, which would include a tenectomy and lengthening of the tendon if indicated. The owner, however, elected conservative management with external coaptation of the left carpus with a palmar fiberglass splint, rather than proceeding with surgical correction at that time. One week after placing the left forelimb splint, the patient presented for a right forelimb lameness. There were no abnormalities noted on physical examination, aside from previously noted moderate carpal valgus and carpal hyperflexion. Despite 6 weeks of external coaptation with the left forelimb splint, no improvement was seen in the flexural deformity or carpal valgus. Therefore, a left tendon lengthening procedure was performed 1 week following splint removal ( - Fig. 3). A bivalve cast was placed on the left forelimb postoperatively and removed 8 weeks after surgery. The left forelimb was recovering well 8 weeks post tendon lengthening with a moderate lameness present after bandage removal (-Fig. 4). Three days later, the right tendon lengthening procedure was performed. And again, a bivalve cast was applied. The patient's activity was restricted for 16 weeks total with instructions to perform short, 5-minute leash-walks three times daily. At 5 weeks postoperatively, the cast was changed to a splint bandage and then removed 8 weeks postoperatively ( $\mathbf{- F i g s .} \mathbf{5}$ and $\mathbf{6}$ ). After bandage removal, the owner was instructed to perform passive range of motion exercises on each operated limb for 6 weeks.

\section{Surgery}

Surgical release of the flexor carpi ulnaris and superficial digital flexor tendons was performed in both forelimbs as staged procedures 8 weeks apart. However, the first procedure for the left forelimb was performed under the same anaesthetic procedure as a revision surgery for recurrence of RMPL. In regard to the surgical procedures for the RMPL revision surgery, the bone within the trochlear groove (previous wedge trochleoplasty) groove was deepened via resection trochleoplasty and was extended further proximally into the femoral metaphysis. A tibial tuberosity transposition was performed and was secured with two Steinman pins and a $20 \mathrm{~g}$ tension band wire. The tibial tuberosity was transposed a little further laterally and distally than previously to correct for patella alta. A medial release was performed using electrocautery. The joint capsule was closed with 0 polydioxanone in a simple continuous pattern. A lateral imbrication was performed using 1 polydioxanone in a modified Mayo mattress pattern on the biceps fascia. Autologous platelet-rich plasma was injected into the stifle joint $(1 \mathrm{~mL})$.

Surgery for the tendons in both forelimbs was otherwise identical: the dog was placed in dorsal recumbency. A linear 


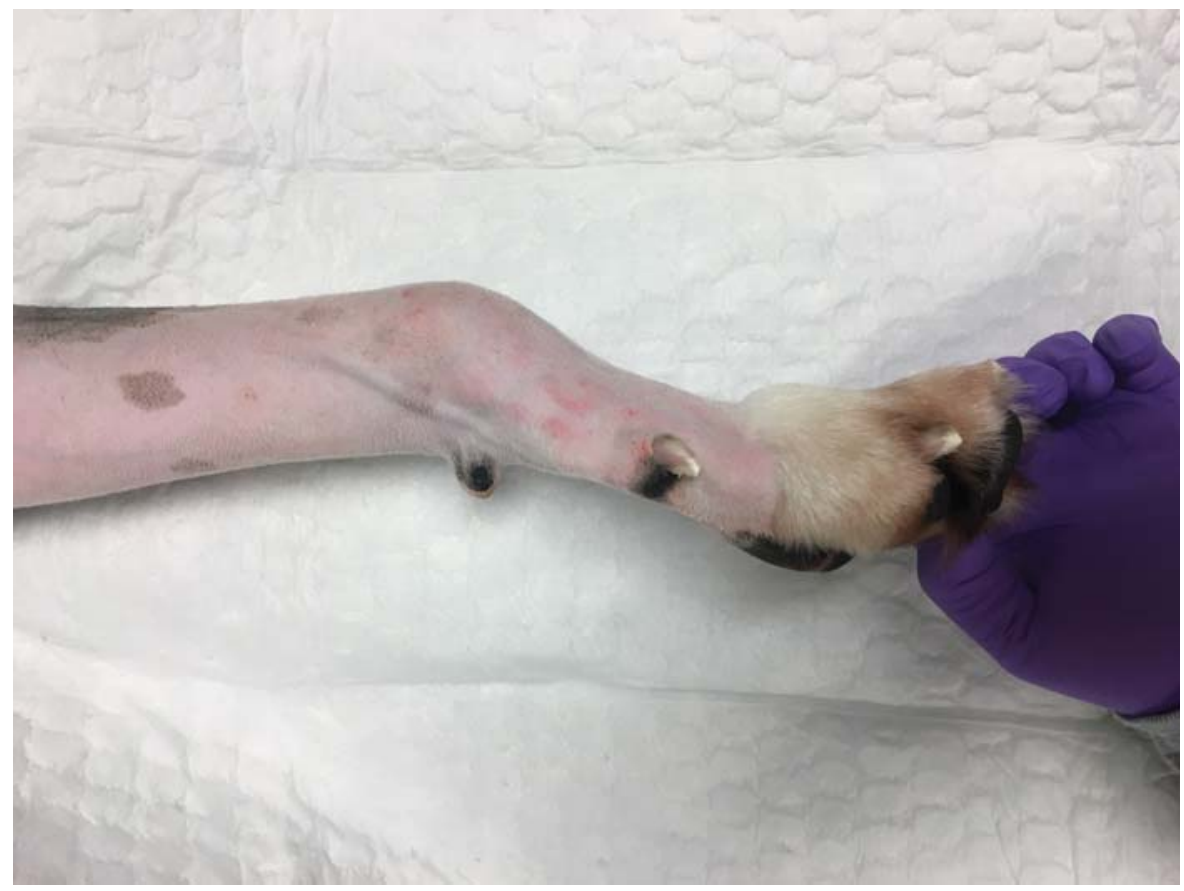

Fig. 3 Preoperative view of the left forelimb with extension of the carpus.

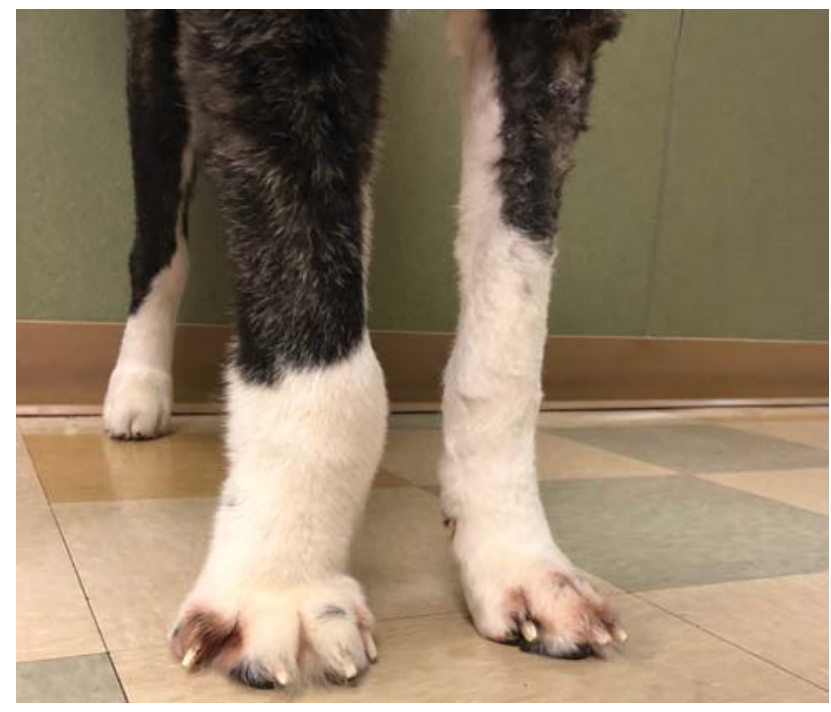

Fig. 4 Frontal view of bilateral forelimbs at 8 weeks postoperatively from the left tendon lengthening procedure.

incision was made at the caudal aspect of the mid-to-distal forelimbs directly over the flexor carpi ulnaris tendons. The skin was retracted with Gelpi retractors, and the flexor tendons was explored. The flexor carpi ulnaris and superficial digital flexor tendons were palpably tight. A z-tenotomy was performed via a longitudinal incision of the flexor carpi ulnaris tendon and tenotomy at each end. A side-to-side anastomosis of the sectioned ends was made using 4-0 Prolene, thereby lengthening the tendon. The same procedure was performed on the superficial digital flexor tendon. Thereafter, the carpus could be fully extended manually and the valgus was significantly improved. Autologous plateletrich plasma was injected into the tendons $(0.5 \mathrm{~mL}$ each) and the carpal joint. ${ }^{10}$ The subcutaneous and intradermal layers

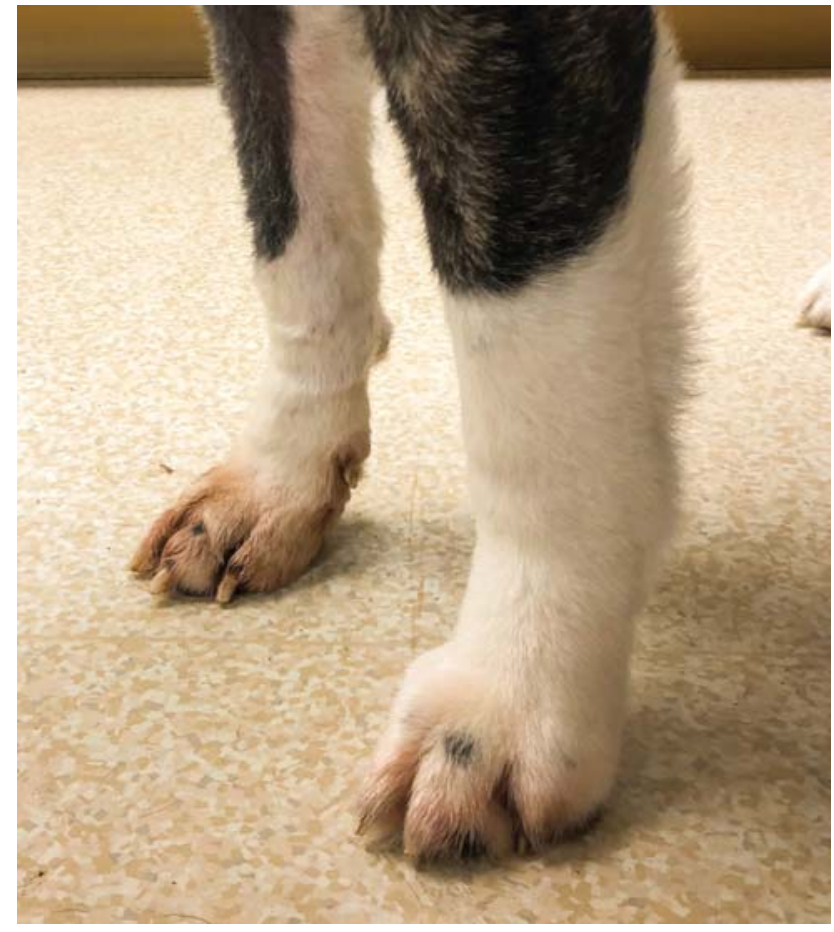

Fig. 5 Lateral view of bilateral forelimbs at 8 weeks postoperatively from the right tendon lengthening procedure.

were infiltrated with $5 \mathrm{~mL}$ of liposomal bupivacaine. The skin was closed routinely. A bivalved cast was applied postoperatively for 5 weeks ( $\mathbf{- F i g s . ~} \mathbf{7}$ and $\mathbf{8}$ ).

\section{Follow-up}

A 6-month follow-up examination revealed no apparent lameness on examination. There was no recurrence of carpal valgus or flexural deformity. Clinically, there were no 


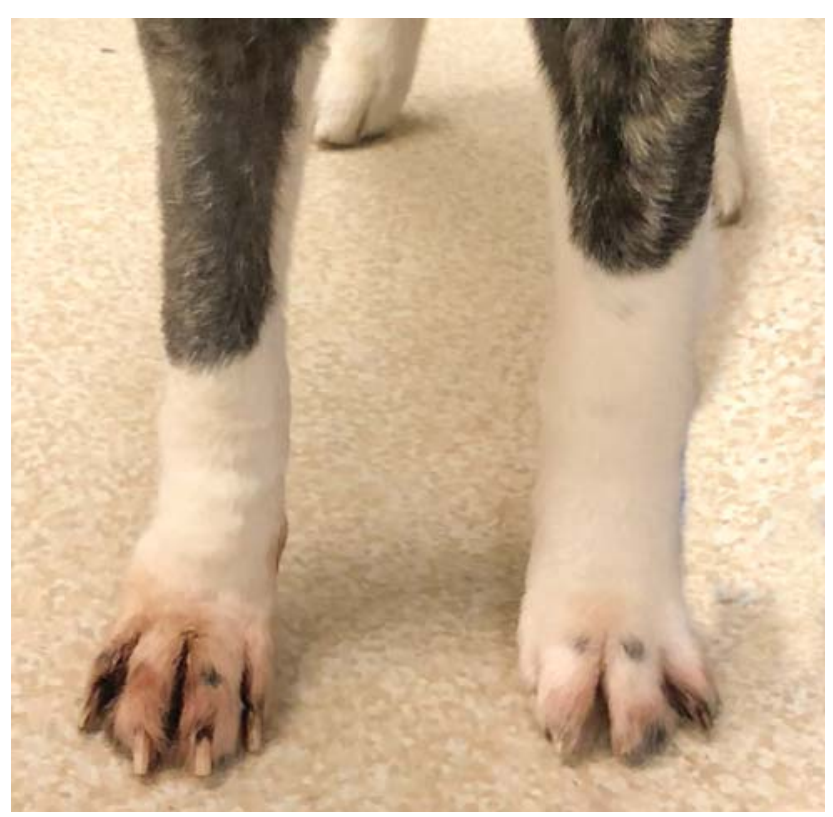

Fig. 6 Frontal view of bilateral forelimbs at 8 weeks postoperatively from the right tendon lengthening procedure.

concerns reported by the owner. The owner was contacted 18 months postoperatively by telephone and reported that the patient had no recurrence of lameness or limb deformity and had normal activity.

\section{Discussion}

The report shows successful surgical management of flexor carpi ulnaris and superficial digital flexor musculotendinous contracture in one dog. A specific underlying cause for this

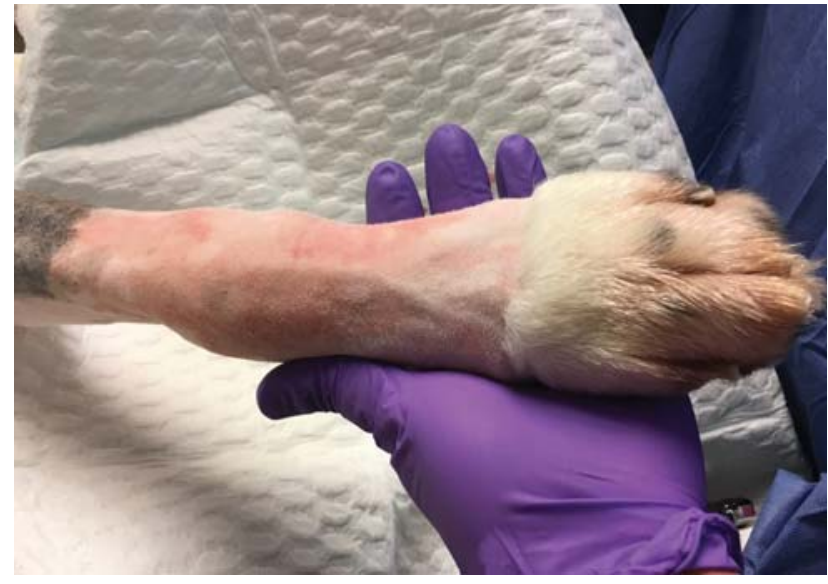

Fig. 8 Postoperative frontal view of the left forelimb revealing marked improvement of carpal valgus.

particular case could not be determined. However, based on the presentation, it was likely congenital or developmental in origin and similar to flexural carpal deformities that occur in young puppies. ${ }^{4}$ Specific treatment for muscle or tendon contracture in young dogs includes medical or surgical management. Generally, juvenile patients have a favourable response to medical management, which often involves splinting and exercise restriction. ${ }^{4-6}$ Pending on the severity or chronicity of the case, as well as initial response to medical therapy, surgical management may be indicated sooner. As described in this case, conservative treatment by splinting of the left forelimb was initially attempted. Since no clinical improvement was seen, surgical correction with z-tenotomy was elected. The z-tenotomy tendon lengthening procedure is one of the most common techniques. ${ }^{11}$ This type of tenotomy

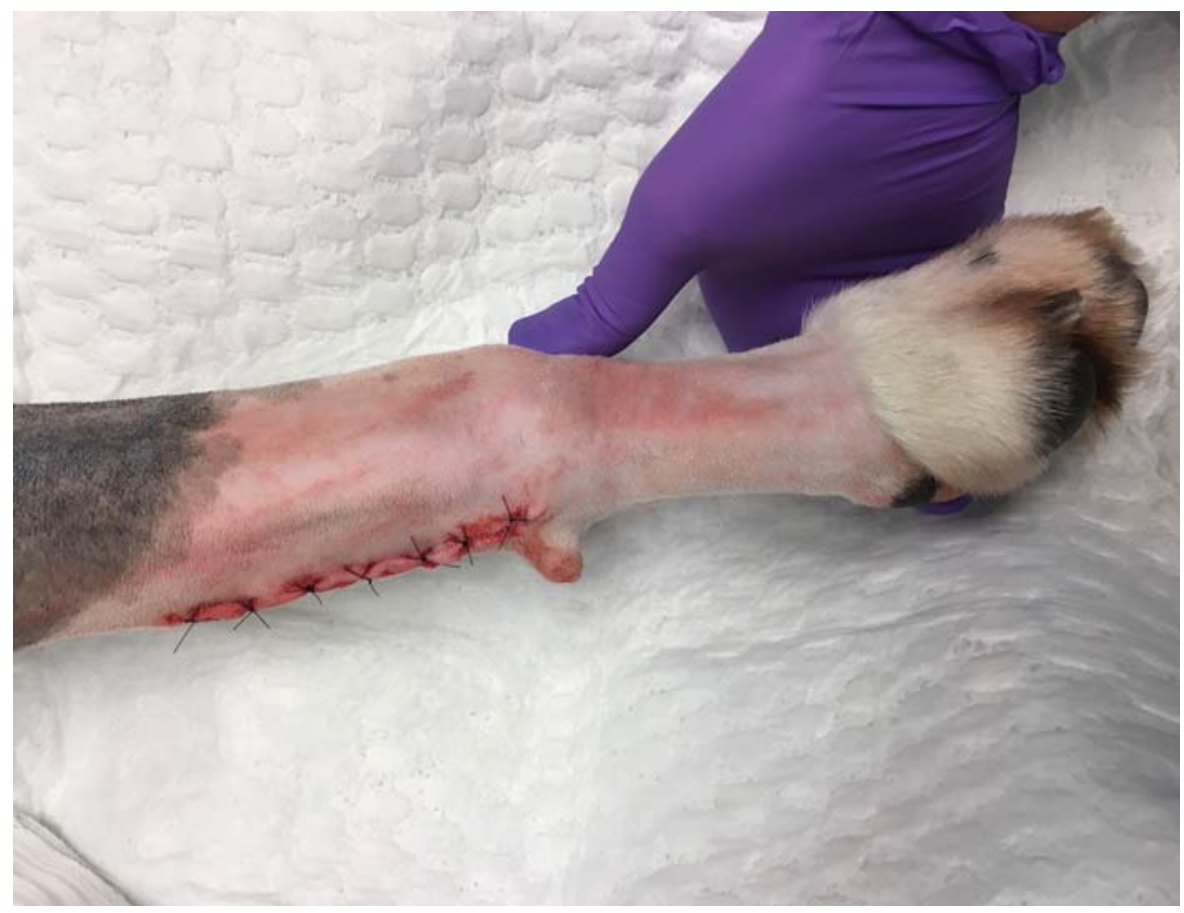

Fig. 7 Postoperative lateral view of the left forelimb revealing marked improvement of carpal valgus. 
involves a longitudinal incision in the affected tendon followed by a transverse incision at each end of the longitudinal incision. The result of the incisions makes an elongated $\mathrm{Z}$ formation. $\mathrm{A}$ suture anastomosis of the tendon ends is performed as the final step. ${ }^{11}$ Other techniques for tendon lengthening include accordion, modified z-tenotomy, oblique section and sliding and the Lange technique. ${ }^{11}$ The $z$-tenotomy approach was utilized in this case because it allows for appropriate tendon healing while minimizing soft tissue trauma and preserving blood supply. As the superficial digital flexor tendon is considered avascular, it relies heavily on intrinsic blood supply during the healing phases. ${ }^{3}$ This type of tenotomy also prevents gap formation, ${ }^{12,13}$ thereby eliminating formation of scar tissue. ${ }^{3}$ Lack of scar tissue optimizes tendon healing due to the decreased strength and higher incidence of adhesion formation. ${ }^{14}$ No surgical complications occurred in this case. Our result suggests that the surgical correction with z-tenotomy lengthening technique for carpal valgus with flexural deformity might be preferable over conservative management with splinting.

\section{Authors' Contributions}

Mark Garneau performed the surgery, provided figures and revised the manuscript. Mikaela Gondolfe compiled patient records and wrote and submitted the manuscript.

\section{Conflict of Interest}

None declared.

\section{References}

1 Blood DC, Studdert VP, Gay CC. Saunders Comprehensive Veterinary Dictionary. 3rd edition. Philadelphia: Elsevier; 2007430
2 Taylor J, Tangner $\mathrm{CH}$. Acquired muscle contractures in the dog and cat. A review of the literature and case report. Vet Comp Orthop Traumatol 2007;20(02):79-85

3 Carmichael S, Marshall W. Muscle and tendon disorders. In: Tobias KM, Johnson SA. eds. Veterinary Surgery: Small Animal. St. Louis: Elsevier; 2012:1127-1134

4 Vaughan LC. Flexural deformity of the carpus in puppies. J Small Anim Pract 1992;33:381-384

5 Altunatmaz K, Ozsoy S. Carpal flexural deformity in puppies. Vet Med 2006;51:71-74

6 Pettazoni M, Mortellaro CM. Flexural deformity in a Dalmatian puppy: a case report and review of literature. Veterinario 2000; 14:33-40

7 Bailey CJ, Culvenor JA. The surgical management of superficial digital flexor contracture in a Great Dane. Aust Vet J 2007;85(12):20-22

8 Mielke B, Kulendra N, Halfacree Z, Kulendra E. Bicipital tendinopathy and carpal valgus following a distant direct thoracoabdominal skin flap in a German Shepherd with necrotizing fasciitis. J Am Anim Hosp Assoc 2018;54(04):226-230

9 Franch J, Bertran J, Remolins G, Fontecha P, Díaz-Bertrana MC, Durall I. Simultaneous bilateral contracture of the infraspinatus muscle. Vet Comp Orthop Traumatol 2009;22(03):249-252

10 Upchurch DA, Renberg WC, Roush JK, Milliken GA, Weiss ML. Effects of administration of adipose-derived stromal vascular fraction and platelet-rich plasma to dogs with osteoarthritis of the hip joints. Am J Vet Res 2016;77(09):940-951

11 Butler HC. Surgery of tendinous injuries and muscle injuries. In: Newton CD, Nunamaker DM, eds. Textbook of Small Animal Orthopaedics. Philadelphia: JB Lippincott; 1985:835-842

12 Clark DM. Tendon injury and repair. In: Bojrab MJ, ed. Disease Mechanisms in Small Animal Surgery. 2nd edition. Philadelphia: Lea \& Febiger; 1993:1079-1082

13 Fahie MA. Healing, diagnosis, repair, and rehabilitation of tendon conditions. Vet Clin North Am Small Anim Pract 2005;35(05): 1195-1211, vii

14 Voleti PB, Buckley MR, Soslowsky LJ. Tendon healing: repair and regeneration. Annu Rev Biomed Eng 2012;14:47-71 